\title{
ASME B89.4.19 standard for laser tracker verification - experiences and optimisations
}

\author{
K.M. Nasr^, A.B. Forbes, B. Hughes, and A. Lewis \\ National Physical Laboratory (NPL), Hampton Road, TW11 0LW Teddington, UK
}

Received: 5 May 2012 / Accepted: 14 June 2012

\begin{abstract}
Laser trackers are becoming the tool of choice for large volume dimensional metrology applications such as the measurement of aerospace assemblies, power plant structures, civil engineering structures and terrestrial transportation vehicles. A laser tracker is a portable coordinate measuring system that tracks a moving target reflector and measures the position of the target in spherical coordinates $(r, \theta, \phi)$. The metrological performance of a laser tracker is influenced by many factors including: compensation for atmospheric effects, thermal expansion of the instrument and its mount, thermal distortion of the workpiece or artefact being measured, the wavelength of the laser radiation, the internal alignment of the gimbal axes and the linearity and alignment of the internal angular measuring scales. The most important of these potential error sources, which fundamentally limit the achievable uncertainty, are the internal mechanical and optical alignments and the quality and alignment of the angular scales. Several national and international standards exist or are in the process of being developed for performance verification of laser trackers. ASME B89.4.19-2006 is one of the established standards used to verify the performance of laser trackers. The main test relies on measuring a known reference length in a variety of configurations and ranges and comparison of the observed error (laser tracker measured length minus reference length) with the specified maximum permissible error (MPE) defined by the manufacturer. The establishment of an ASME B89.4.19 laser tracker verification facility at NPL is introduced. We highlight the importance of tracker verification and discuss the error sources, which contribute to the tracker measurement uncertainty. Some initial results obtained using this new facility are presented.
\end{abstract}

Keywords: Large volume dimensional metrology; laser trackers; ASME B89.4.19-2006; performance verification; uncertainty; spherical coordinate measurement systems; portable measurement systems

\section{Introduction}

Laser trackers have become popular measuring tools for users needing to make precision measurements of items that are simply too large to be measured by Coordinate Measuring Machines (CMM) or of items which are non-portable.

Typical applications of large volume dimensional metrology using laser trackers include the measurement of aerospace assemblies, power plant structures, civil engineering structures and terrestrial transportation vehicles.

A laser tracker (Fig. 1) is a portable coordinate measuring system that tracks a moving target reflector and measures the position of that target in spherical coordinates $(r, \theta, \phi)$. The radial distance or range component is typically measured by an interferometer (IFM) or an Absolute Distance Meter (ADM). The IFM or ADM laser beam is steered to track the moving target, usually a Spherically Mounted Retroreflector (SMR),

^ Correspondence: karim.nasr@npl.co.uk by a motorised gimbal mechanism. Angle encoders on the mechanism determine azimuth and elevation angles of the target.

Typical models include trackers from manufacturers such as API, Leica and Faro, which incorporate either ADM and/or IFM modes of operation (e.g., API T3/Radian, Leica 901/401, FARO ION). The typical range of operation is from $30 \mathrm{~m}$ to above $120 \mathrm{~m}$.

As with any metrology tool, a laser tracker needs to undergo periodic performance verification.

The most commonly used standard for laser tracker performance verification is ASME B89.4.19-2006 [1]. Other standards such as the VDI/VDE 2617 Part 10 and the ISO 10360- Part 10 (draft) are in different stages of development $[2,3]$.

In Section 2, a brief overview of the laser tracker verification procedure is given. More specific details of the B89.4.19 volumetric and two face tests are presented in Sections 3 and 4. Some typical results for the B89.4.19 tests performed at NPL for laser tracker verification are 


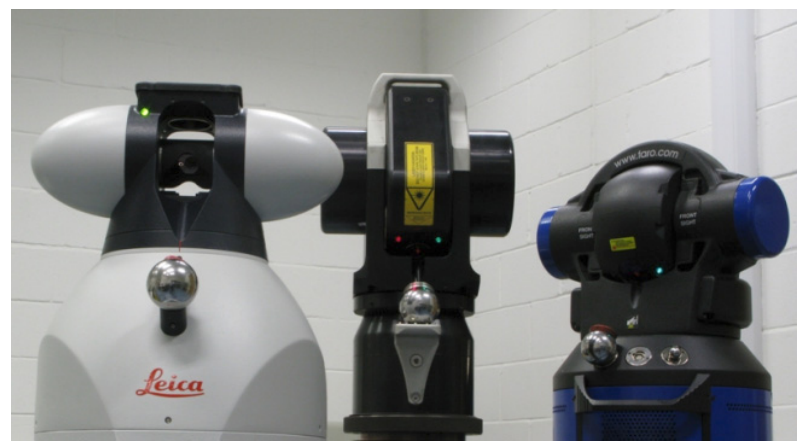

Fig. 1. Examples of laser trackers.

presented in Section 5. An NPL alternative methodology currently being tested and verified is introduced briefly in Section 6 . The main advantage of this new methodology is a considerable reduction in testing time and giving more insight into why a laser tracker might not have passed a B89.4.19 test. Finally, Section 7 concludes the paper.

\section{Overview of laser tracker verification process and the ASME B89.4.19 standard}

The verification processes at NPL include the following main steps:

- laser wavelength calibration;

- environmental sensors (temperature, pressure and humidity) calibration;

- specific manufacturer calibration procedures; and

- laser tracker performance verification using ASME B89.4.19.

The first part of the verification for trackers that have an IFM fitted, is to determine laser frequency using a direct beat-frequency comparison against an iodine-stabilised primary laser that is an internationally accepted realisation of the metre $[4,5]$.

Next, the performance of the air temperature, air pressure and part temperature probes are verified by comparison against similar sensors which have been previously calibrated by NPL.

The distance measuring capability of the tracker is tested over a range of $30 \mathrm{~m}$ (ranging test) to verify the fringe counting performance of the laser interferometer (if fitted) as well as the accuracy of the absolute distance meter. A carriage is mounted on rails on a tape bench and the position of the carriage is measured using an NPL displacement interferometer. Additional sensors are used to measure air temperature, pressure and humidity in order to derive a refractive index correction to the laser vacuum wavelength. The NPL interferometer laser and the air sensors are previously calibrated with traceability to national standards. The carriage is moved to a series of locations along the linear rail. At each location, the carriage is stopped and held stationery whilst the laser tracker and NPL laser interferometer are read. The data from the laser

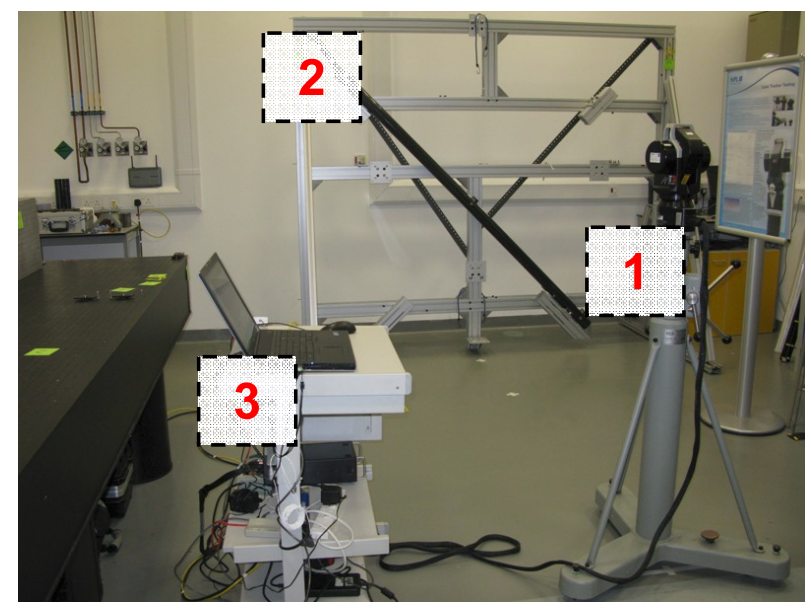

Fig. 2. ASME B89.19.4 tracker verification service at NPL showing (1) tracker, (2) rig and (3) laptop used for data acquisition and post processing.

tracker are obtained by acquiring a series of points using Spatial Analyzer [6] software. A single measurement run consists of a measurement at the starting location, near to the laser tracker, followed by measurements at carriage locations at increasing distance from the start location, followed by repeated measurements at the same nominal locations as the carriage is returned towards the laser tracker. The final measurement in each sequence is a re-measurement at the start location. The environmental conditions are recorded at the start and end of each measurement run. As well as making linear range measurements, point-to-point distance measurements are calculated for all the points to ensure that any alignment errors between the laser tracker and the reference interferometer are negligible. Three measurement runs are performed, as required by ASME B89.4.19.

The ASME B89.4.19-2006 standard requires several tests that rely on measuring a known reference length (artefact) and comparing the observed error (laser tracker measured length minus reference length) with the specified maximum permissible error (MPE) given by the manufacturer. The main artefact (or reference length) is specified to be at least $2.3 \mathrm{~m}$ long. All tests are done in IFM and ADM modes. NPL recommends starting with the easier ADM test and checking whether the resulting ADM volumetric performance also satisfies the IFM volumetric MPE specifications. If this is the case, then there is no need to do the IFM tests because the performance of the tracker should always be better in IFM mode (assuming the IFM laser has been correctly calibrated). The tests are designed to exercise the tracker measurement system over a wide range of angles and distances similar to those encountered in real world use.

The NPL service (Fig. 2) utilises the seven tests prescribed in ASME B89.4.19 as follows:

- horizontal length measurement system test (nine configurations); 
Table 1. Main B89.4.19 volumetric test configurations.

\begin{tabular}{ccccc}
\hline Mode & Ranges $(\mathrm{A}=2.3 \mathrm{~m})$ & Angles & Orientation & Targets \\
\hline ADM, IFM & $0.1 \mathrm{~A}=0.23 \mathrm{~m}$ & Any & H only & 2 \\
ADM, IFM & $1.2 \mathrm{~A}=2.76 \mathrm{~m}$ & $0^{\circ}, 90^{\circ}, 180^{\circ}, 270^{\circ}$ & $\mathrm{H}, \mathrm{V}, \mathrm{RD}$ and LD & 2 \\
ADM, IFM & $2.7 \mathrm{~A}=6.21 \mathrm{~m}$ & $0^{\circ}, 90^{\circ}, 180^{\circ}, 270^{\circ}$ & $\mathrm{H}, \mathrm{V}, \mathrm{RD}$ and LD & 2 \\
\hline
\end{tabular}

Total number of measurements $=3 \times(2$ modes $) \times(2$ ranges $) \times(4$ angles $) \times(4$ orientations $) \times(2$ targets $)+3 \times(2$ modes $) \times$ $(1$ range $) \times(1$ angle $) \times(1$ orientation $) \times(2$ targets $)=(192+6) \times 2=396$.

Abbreviations: H: Horizontal, V: Vertical, RD: Right Diagonal, LD: Left Diagonal.

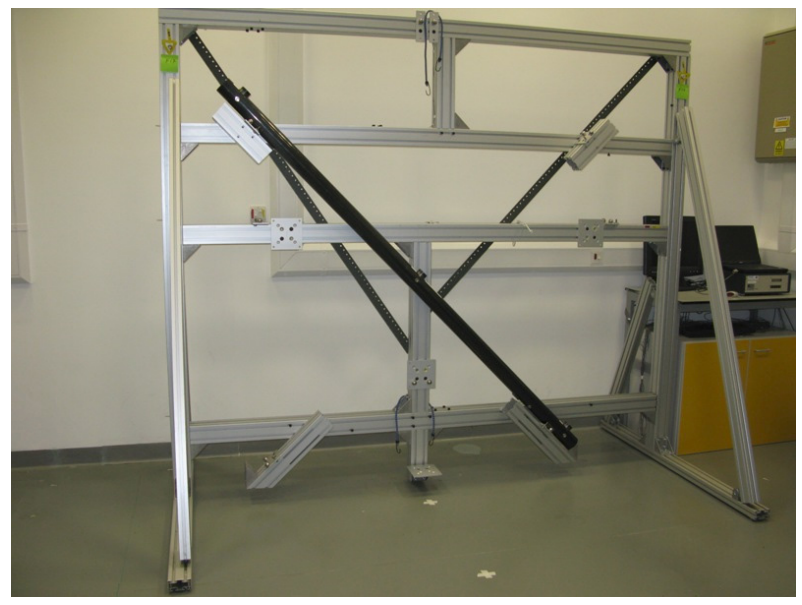

Fig. 3. B89.4.19 artefact and support rig.

- vertical length measurement system test (eight configurations);

- right diagonal length measurement system test (eight configurations);

- left diagonal length measurement system test (eight configurations);

- two-face system test measurement (twelve configurations);

- ranging tests (IFM and/or ADM) up to $30 \mathrm{~m}$; and

- user selected volumetric tests (two configurations).

The above configurations test the horizontal and vertical angle encoder scale errors and eccentricities, as well as the distance and displacement measuring systems.

The SMR used in the tests can be 0.5 inch, $7 / 8$ inch or 1.5 inch diameter depending on the manufacturer or customer preference. The same SMR is used throughout, for all tests. The duration of the long-range test is one day and a further two to three days are required for the laser and sensor calibrations. The duration of a full B89.4.19 test consisting of a volumetric test and a two-face test is two days.

\section{B89.4.19 main volumetric test}

The length measurement system tests and the two-face system tests are performed at NPL using a reference length artefact (Fig. 3) as specified in ASME B89.4.192006 Section 6.2.1 (a). The artefact consists of a $2.5 \mathrm{~m}$ long, $76 \mathrm{~mm}$ outer diameter carbon fibre tube mounted on a support frame. Two 1.5 or 0.5 inch kinematic nests are fixed on the outer surface of the tube at a nominal separation of $2.31 \mathrm{~m}$.

The measurand is the point-to-point separation of the nests. The artefact is calibrated by using a verified interferometer of the laser tracker to measure the inter-nest separation, whilst the artefact is aligned such that the measurand lay on a radial line from the tracker. This alignment is made to within 20 arc minutes. The artefact is calibrated at the start and end of the B89.4.19 volumetric measurements. Each calibration is the average of ten measurements. Either the NPL-owned tracker or the tracker under test may be used, provided that the tracker's IFM laser has been pre-verified for wavelength value and ranging capabilities. The NPL artefact calibration was checked using a coordinate measurement machine (CMM) and was found to be in good agreement with the laser tracker calibration.

The following general procedures are implemented during the tests:

- the tracker should be clamped to prevent it from moving during measurements;

- the SMR should be positioned in approximately the same orientation relative to the measurement laser beam to minimise the influence of geometric errors when repeating the tests;

- environmental conditions (e.g., temperature and pressure must be monitored and recorded);

- for any measurement which fails to satisfy the MPE requirements, the reference length is first checked then the failed measurement is repeated a maximum of five times and the worst result is then substituted for the failed measurement.

The different configurations of the main test are shown in Table 1. An illustration of the main test configurations is shown in Figure 4.

To reduce the time of tests, the measurement order of the two targets (A \& B which are repeated three times per configuration as shown above) can be AB-BA-AB instead of $\mathrm{AB}-\mathrm{AB}-\mathrm{AB}$.

Further time optimisation can be performed by moving the tracker first to the different ranges for the same artefact position. This applies to the case when it is easier and faster to move the tracker rather then repositioning the heavy artefact to a new orientation. Hence, performing an $(r \times o)$ rather than an $(o \times r)$ tests with $\mathrm{r}$ being the different tracker ranges and o being the artefact orientation. 
Table 2. Two face test configurations.

\begin{tabular}{cccccc}
\hline Mode A & Mode B & Ranges: D $(\mathrm{A}=2.3 \mathrm{~m})$ & Angles & Orientation & Targets \\
\hline Front, Back & ADM & Less than $1 \mathrm{~m}$ & $0^{\circ}, 90^{\circ}, 180^{\circ}, 270^{\circ}$ & $\mathrm{V}$ & 3 \\
Front, Back & ADM & $3 \mathrm{~m}$ & $0^{\circ}, 90^{\circ}, 180^{\circ}, 270^{\circ}$ & $\mathrm{V}$ & 3 \\
Front, Back & ADM & $6 \mathrm{~m}$ & $0^{\circ}, 90^{\circ}, 180^{\circ}, 270^{\circ}$ & $\mathrm{V}$ & 3 \\
\hline
\end{tabular}

The total number of two face measurements $=(3$ times $) \times(2$ modes $\mathrm{A}) \times(1$ mode $\mathrm{B}) \times(3$ ranges $) \times(4$ angles $) \times$ $(3$ targets $)=216$.

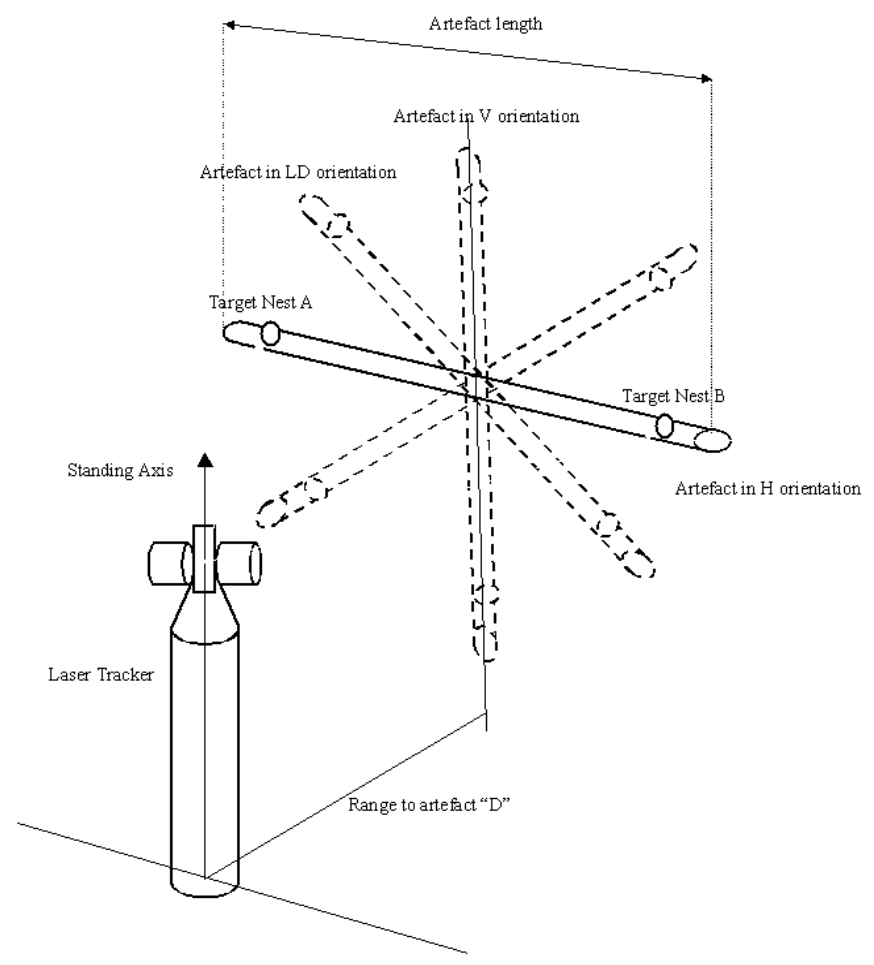

Fig. 4. Illustrations of the four B89 main test configurations.

NIST suggested [7] two specific user defined configurations, which are also recommended by NPL as follows:

(1) asymmetrical diagonal test with the tracker and one of the targets in the same plane rather than symmetric and at a range of $0.5 \mathrm{~m}$;

(2) horizontal length above tracker (e.g., tracker on the floor and pointing upwards towards the horizontal artefact.

The total number of volumetric tests equates to $(198+$ 12) $\times 2=420$.

\section{Augmented two-face test}

The two-face test is performed using the same carbon fibre artefact that is used for the length measurement volumetric tests. An additional kinematic nest is fixed at the approximate centre of the tube, mid-way between the

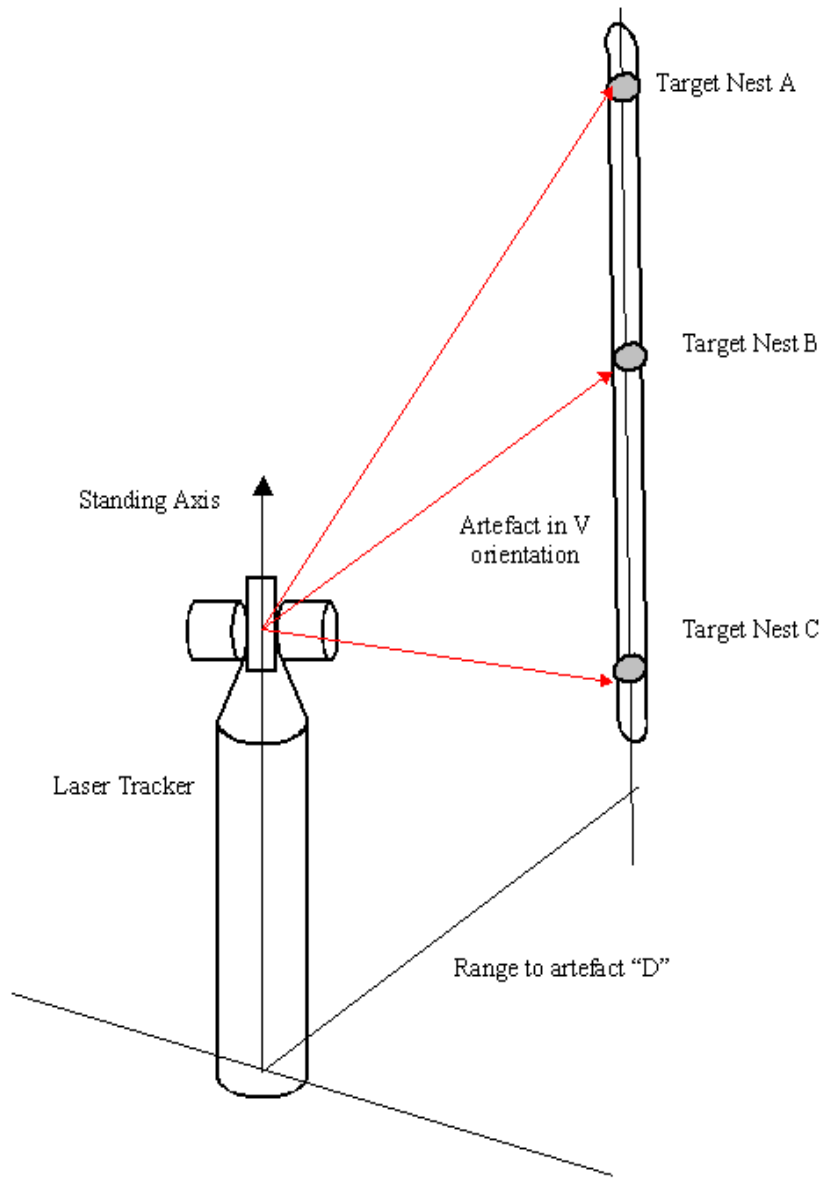

Fig. 5. Illustration of the two-face test configuration.

two nests used for the length measurement system tests. The artefact is supported on the same support frame, in the same orientation as used for the vertical length measurement system tests. The two-face test is performed using only the ADM. The test configurations are shown in Table 2 and illustrated in Figure 5. These tests are also repeated three times. The B89.4.19 standard specifies the measurements of three SMR points in each mode (front and back) and calculating the Euclidian distance between the same points in each mode then taking the largest value to compare with the MPE specifications. 


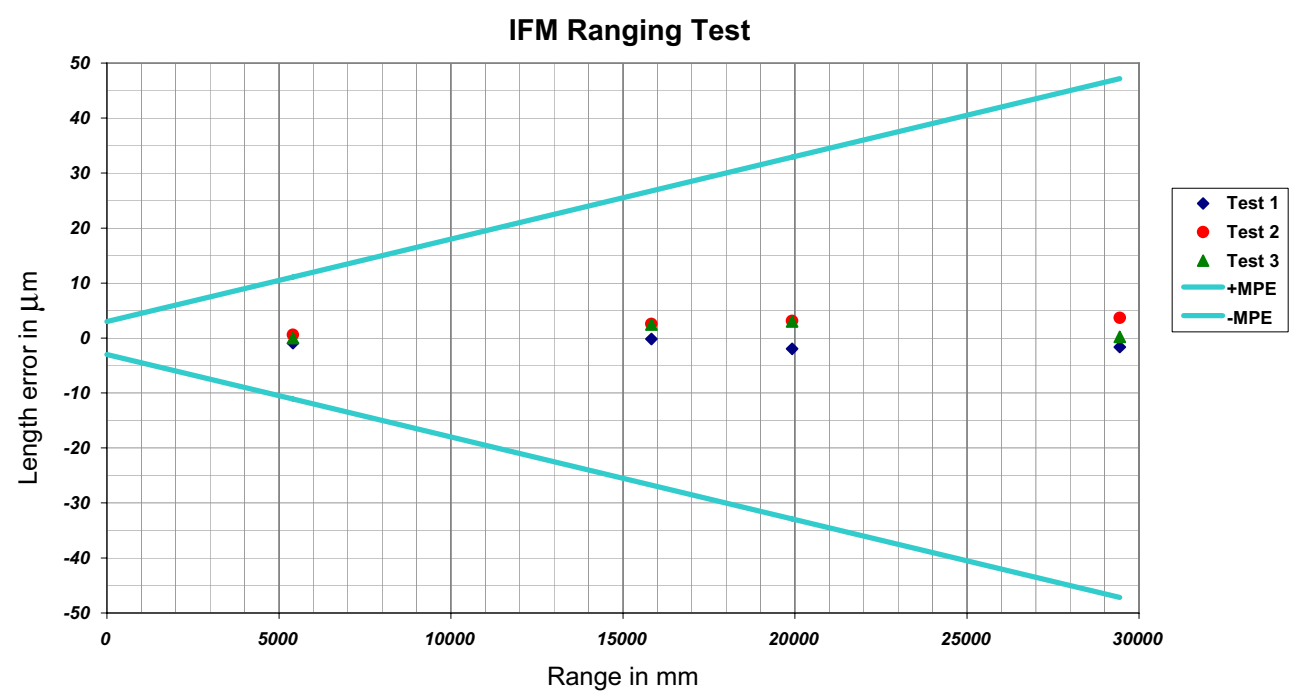

Fig. 6. Interferometer ranging test results.

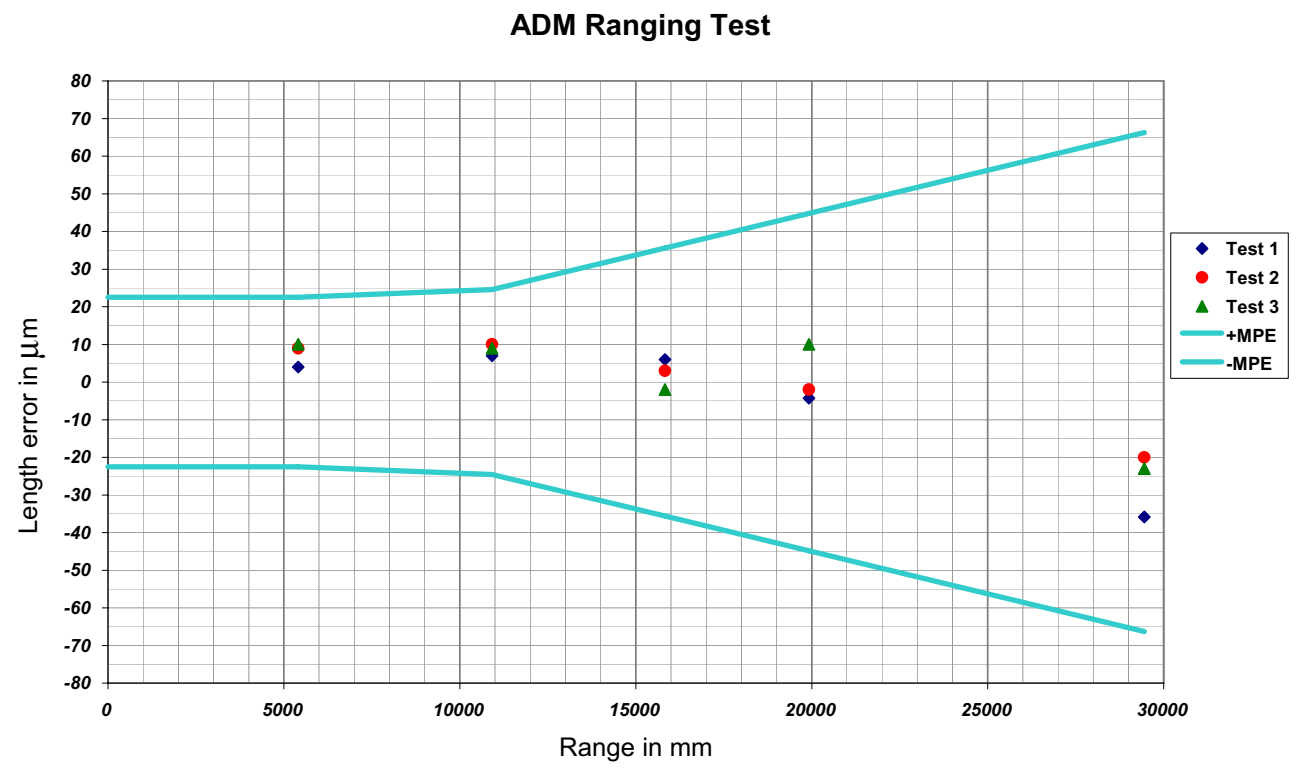

Fig. 7. ADM ranging test results.

\section{Example results}

Typical results from long ranging interferometer tests are summarised in Figure 6 and those from the ADM ranging tests in Figure 7. Typical B89.4.19 volumetric length measurements test of a laser tracker conducted at NPL are summarised in Figure 8 (results obtained using the interferometer mode) and Figure 9 (results obtained using the ADM mode). The results from a typical two-face test are summarised in Figure 10.

The results show that the tested tracker complies with the manufacturer's MPE window for all measurements. MPE values are individually calculated due to different ranges to each specific target. It is necessary to obtain from the manufacturer an explicit statement of the MPE calculation parameters as these are often not detailed enough in the manuals. The ranging performance of the IFM is better than that of the ADM, which is to be expected. The spread in tests 1,2 and 3 is mainly due to random effects (e.g., atmospheric environment and operator skill).

\section{NPL network based calibration methodology}

A novel network-based laser tracker testing/calibration methodology, demonstrated by NPL [8] has received great interest from the laser tracker manufacturers and industrial community. The method is approximately eight times faster than existing methods in the published standard (ASME B89.4.19-2006) and simple enough to be performed by end users, with no specialist equipment. Furthermore, the network-based test gives detailed 
IFM Volumetric Tests

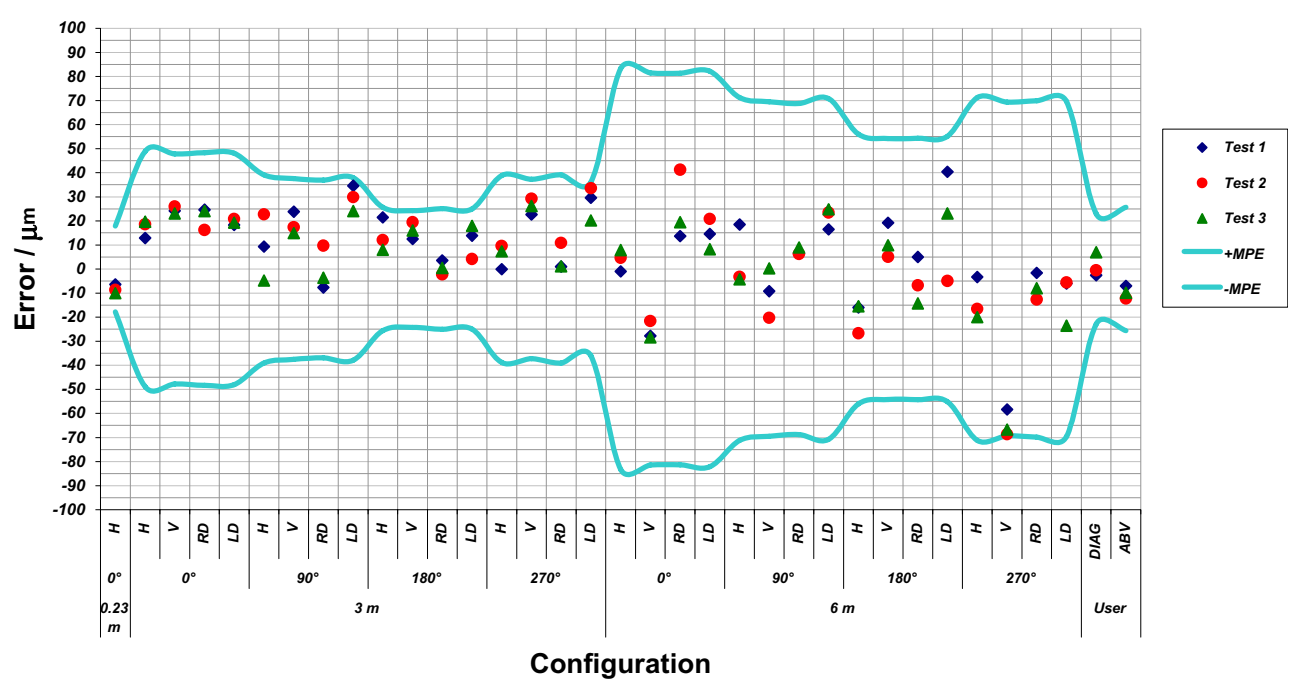

Fig. 8. Length measurement system test results (interferometer mode).

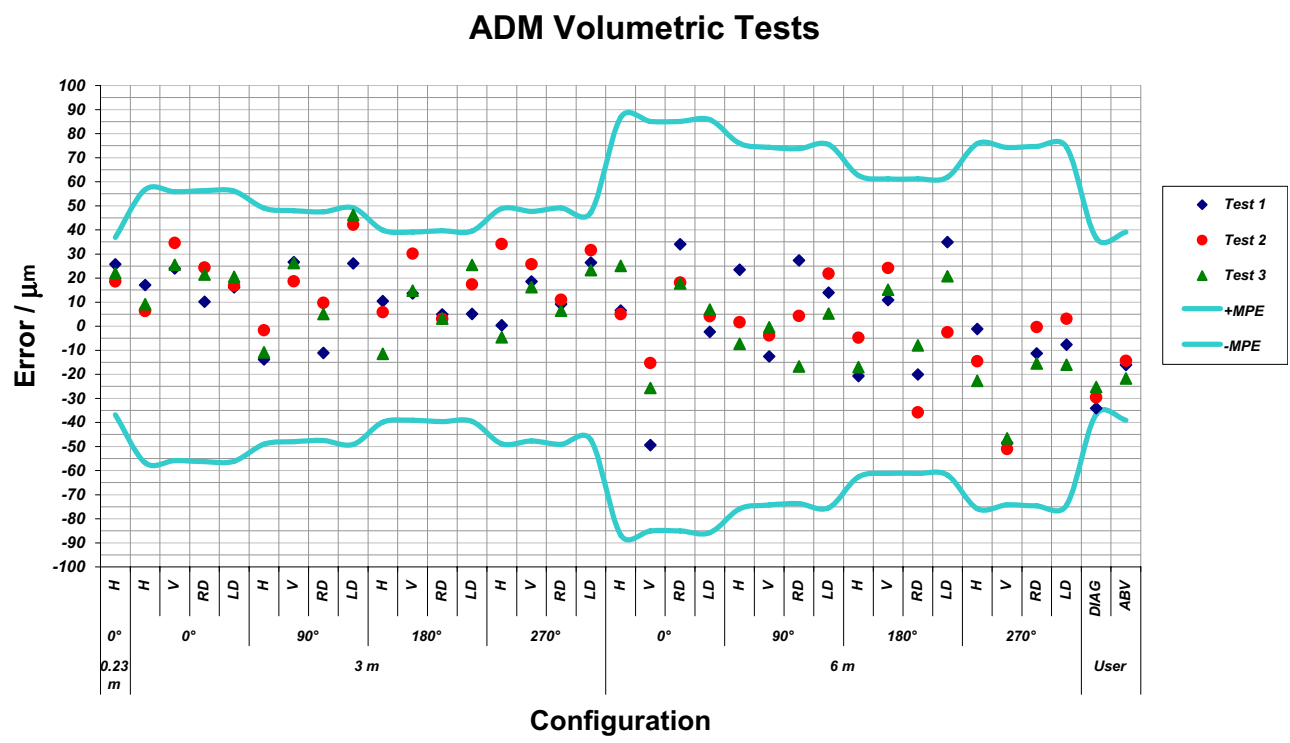

Fig. 9. Length measurement system test results (ADM mode).

quantitative information about the tracker, as well as rigorous uncertainties for the results.

The test can be used not only for performance verification, but also for periodic monitoring of tracker sub-systems and drift/wear of mechanics. The uncertainty evaluation can be used to predict task-specific performance of the tracker, enabling users to judge fitness for purpose.

The NPL network test involves the measurement of a network of fifteen nests at five independent tracker positions/rotations. An in-house developed multi-lateration software application uses the collected network data to solve a nonlinear optimisation problem to obtain the correct positions of the tracker, the location of network nests and the error correction factors to be applied to the internal tracker error map. This methodology is currently being verified through rigorous tests and will be offered as an alternative to the B89.4.19 test. Our intention is to process the data from the NPL network test so that it can be used to generate 'virtual test results' specific to either the VDI/VDE 2617 or ASME B89.4.19 formats.

\section{Conclusions}

An overview the ASME B89.4.19 service for laser tracker performance verification at NPL has been described. The main details of the standard together with descriptions of the different tests were given. Typical example results from the main volumetric test; the two-face tests and ranging tests were also presented.

The current lengthy B89.4.19 verification process is a pass or fail test. An alternative network based testing 
Two face Tests

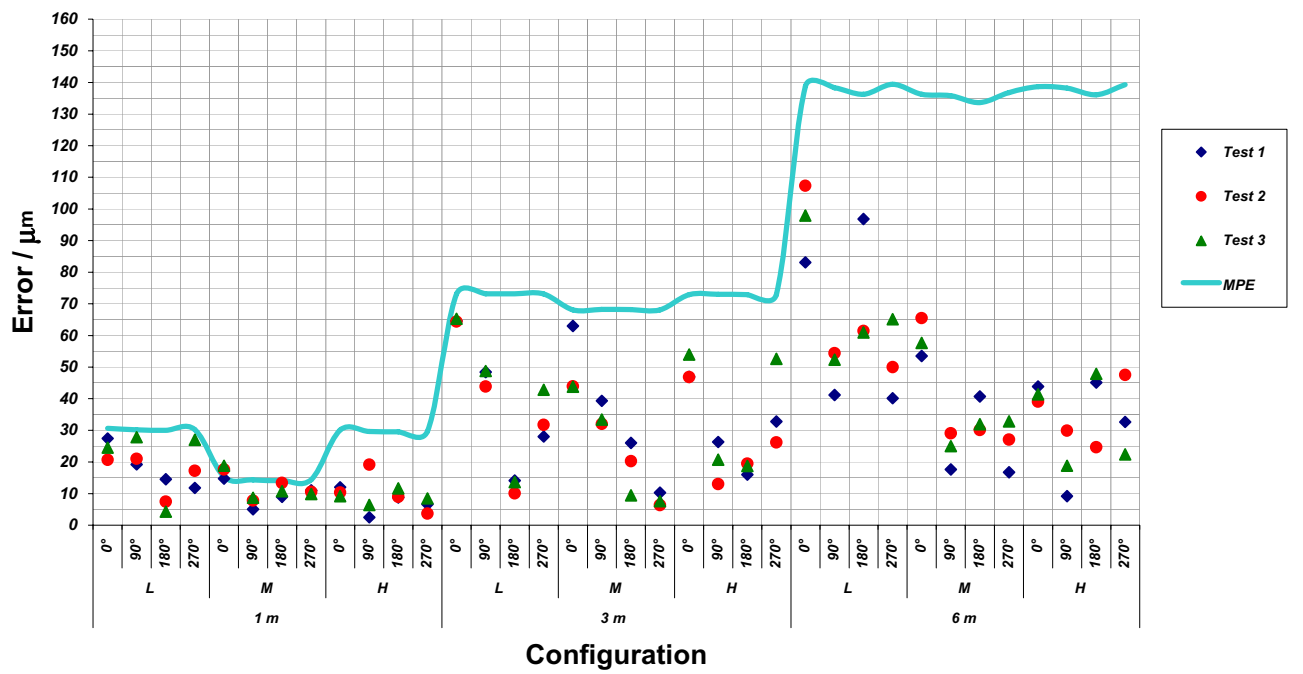

Fig. 10. Two face system test results (ADM).

methodology is currently being tested and verified at NPL with the aim of reducing considerably the measurement time and giving detailed quantitative information about the tracker, as well as rigorous uncertainties for the results.

\section{References}

1. ASME B89.4.19-2006: Performance Evaluation of Laser Based Spherical Coordinate Measurement Systems, ASME, November 2006

2. VDI/VDE 2617 Blatt10/Part 10: Genauigkeit von Koordinatenmessgeräten, Kenngrößen und deren Prüfung, Annahme- und Bestätigungsprüfung von Lasertrackern; Accuracy of coordinate measuring machines, characteristics and their checking, acceptance and reverification tests of laser trackers; Verein Deutscher Ingenieure/Verband Der Elektrotechnik Elektronik Informationstechnik, January 2011
3. ISO 10360 - Part 10 (draft): Geometric product specifications (GPS) - Acceptance and reverification tests for coordinate measuring systems (CMS) - Part 10: Laser Trackers for measuring point to point distances, (Draft) May 2010

4. Documents concerning the definition of the metre, Metrologia 19, 163-177 (1984)

5. T.J. Quinn, Mise en pratique of the definition of the metre, 30, 523-543 (1992)

6. www.kinematics.com/products/spatialanalyzer/overview. html, last accessed October 2011

7. B. Muralikrishnan, D. Sawyer, C. Blackburn, S. Phillips, B. Borchardt, W.T. Estler, ASME B89.4.19 performance evaluation tests and geometric misalignments in laser trackers, J. Res. Natl. Inst. Stan. 114, 21-35 (2009)

8. B. Hughes, W. Sun, A. Forbes, A. Lewis, D. Veal, K. Nasr, Laser tracker error determination using a network measurement, Meas. Sci. Technol. 22, 045103 (2011) 